\title{
Interval Training with Blood Flow Restriction on Aerobic Performance among Young Soccer Players at Transition Phase
}

\author{
Ali Reza Amani ${ }^{1}$, Hassan Sadeghi ${ }^{2}$, Taher Afsharnezhad ${ }^{1}$
}

\begin{abstract}
Affiliations: 'Shomal University, Sport Science Faculty, Amol, Iran, ${ }^{2}$ University Putra Malaysia, Department of Sport Studies, Selangor, Malaysia

Correspondence: A.R. Amani, Shomal University, Department of Sport Science, Mazandaran Province, 5 Haraz Road, Amol, Iran. E-mail: a.amani@ijaep.com
\end{abstract}

ABSTRACT The purpose of current study is investigating effect of Blood Flow Restriction Interval Training on aerobic performance among young football players during transition phase of periodization. Twenty eight young male football players were recruited in this study. Subjects were randomly divided into three groups: control, Normal Interval without Kaatsu, and Interval with Kaatsu. Exercise protocol was based on aerobic interval at 400 meters with maximum effort for 3 sets in per sessions (First week) and 4 set (Second week). The results shown that there is a significant difference in aerobic power between groups $p<0.05$. Post hoc comparisons indicated that the mean of aerobic power was significantly different between all three groups. It has been shown, there is a significant difference in rate of perceived exertion between groups at the $\mathrm{p}<0.05$. Post hoc comparisons indicated that the mean of RPE was significantly different between groups. This study has been shown that aerobic power and RPE was improved by both normal and BFR interval method. We also found that there is not significantly difference in NIWK and IWK group at rate of perceived exertion. This results may be explained by reducing intensity of exercise by subjects during exercise with BFR in cause of pain in their legs during exercise. The result of current study suggest that Intermediate-intensity, interval training with BFR improves aerobic capacity and RPE concurrently in young football players and prevent decline of $\mathrm{VO} 2 \mathrm{max}$ at result of retraining at transition phase.

KEY WORDS aerobic power, maximum oxygen uptake, blood flow restriction training, interval training

@MJSSMontenegro

INTERVAL TRAINING WITH BLOOD FLOW RESTRICTION ON AEROBIC PERFORMANCE http://mjssm.me/?sekcija=article\&artid=156

\section{Introduction}

Endurance performance is one of the most important aspect of successfully at football (Helgerud, Engen, Wisløff, \& Hoff, 2001). Players with high level of aerobic and endurance ability may cover tactics and techniques tasks for achieve the best results during competitions (Clemente et al., 2013). Ability to run more for players or at the other hand distance cover during each full time competition significantly influenced by aerobic capacity and endurance performance. Results of several investigations regarding to aerobic performance and its important variables as well as maximum oxygen uptake it has been shown that aerobic capacity may enhance by the several methods by using aerobic energy system training such as interval training, high intensity interval training and also long slow distance training (LSD)(Gibala et al., 2006; Helgerud et al., 2007; Weston et al., 1996). Lack of aerobic performance during transition phase at football player could be with less acceleration by some exercise routines as well as long slow distance or aerobic interval training. However, a major problem with this kind of exercise routines is long duration of exercise protocol. Traditionally load of interval training could be adjusting by variables as well as time (speed) and distance at per repetition, number of sets, intensity of training base of heart rate, recovery time and frequencies of training sessions (Bangsbo, 2003). Several adaptation influences on human body to improve ability of aerobic energy system as well as mitochondria density, respiratory capacity, capillary densities and etc. (Rivera-Brown \& Frontera, 2012). Aerobic training at any target heart rate maybe improve variables influenced on aerobic capacity. Level of 
adaptations and time to reach at degree of adaptation according to training objectives are determining type of training which coaches and athletes may choose. For aiming cardiovascular improvement they choose low level of zone at target heart rate (below the 60\% maximum heart rate) and for developing endurance performance they use normally high level zone at target heart rate (upper $80 \%$ maximum heart rate) (Benson \& Connolly, 2011; Gilman, 1996). Blood flow restriction training (BFR) is a cutting-edge, exercise method that allow athletes to gain training objectives with low level of intensity (but most of time with increasing volume of training). This type of training normally called as KAATSU (means blood flow restriction training) training developed by Dr. Yoshiaki Sato (Cook, Clark, \& Ploutz-Snyder, 2007).

Recently several studies have been shown restricting blood flow during resistance exercise termed KAATSU Training improved some aspects of body composition as well as muscle mass and strength significantly (Abe et al., 2010). Increasing one repetition maximum and muscle size by using KAATSU training method have been reported by several researches (Cook et al., 2007). The main mechanisms by the blood restriction training is thought to stimulate improving muscle mass is stimulates of fast-twitch fiber recruitment (FT), and increased protein synthesis (mTOR) pathway and stimulate of growth factors at anabolic pathways (Loenneke, Wilson, \& Wilson, 2010) and increased protein synthesis through the GH-IGF-1 axis. Improving anaerobic energy system at result by this type of training combined with high intensity interval training has been reported by the new study (Behi, Fahey, Afsharnezhad, \& Amani, 2017) increasing glycogen content of muscle cells, increasing creatin phosphate synthesis and improving muscle function are the main reasons to improving anaerobic performance. Improving aerobic and anaerobic performance at the same time with the same type of exercise or least reducing conflict effect by these type of training is important. In parallel with muscular fitness at result by KAATSU training in combined with aerobic exercises has been shown significant improvement at maximum oxygen uptake and maximum heart rate. Ischemia enhanced adaptation to aerobic and endurance exercise by increasing muscle glycogen content and variables related to maximum oxygen uptake (Park et al., 2010).

Increasing muscle capillary density at resulted by blood restriction training has been shown at recent researches. Increasing capillary density will increase ability of muscle cells to receive more oxygen (Kirkendall \& Garrett, 1998) and then increasing aerobic metabolism. Increasing vascular endothelial growth factor (VEGF) and angiogenesis has been reported at recent studies. VEGF and angiogenesis increase by result of traditional interval training but has been shown significant increase by result of VEGF and angiogenesis by blood restriction training (Evans, Vance, \& Brown, 2010; Presta et al., 2005). Rate of perceived exertion is one of the variables that may effect on endurance performance. At the workload with same intensity athletes with high level of aerobic fitness reported with lower points of RPE (Lower point means reducing fatigue at the same situation) (Scherr et al., 2013).

Debate continues about the best strategies for improving aerobic performance after competition phase at football players. Blood restriction method was applied at several research regarding to strength and muscular hypertrophy but so far this method has only been applied at the current research to improve aerobic performance. Researchers at the following study hypothesis that blood restriction training may influence on human performance over the anaerobic and strength variables. This type of training may effect on aerobic performance for developing aerobic energy system at short and limited duration of time. Developing aerobic and endurance capacity at short duration of time and also reducing confliction effect by the aerobic-strength training at the same time is vital aspect of athletes conditioning program. Thus, the purpose of the current study was to investigate the influence of Blood Restriction Aerobic Interval Training on aerobic performance and rate of perceived exertion among football players.

\section{Methods}

Participation and design of study

This study was an experimental research design. This study was in agreement with the principle of Helsinki Declaration and approved by the ethical committee in the Somal Medical University, Iran. Twenty eight young male soccer players were recruited in this experimental research (age: 23.89 \pm 2.26 years; weight: $73.04 \pm 3.90$; height: 176.1 \pm 4.05 ). All subjects fill up informed consent form before participants to study. The inclusion criteria of participants were: football player, able to do interval exercise, has at least 7 year football experience. And the exclusion criteria also were: football player with chronic injury, player who stop football player since 6 month ago. Subjects were randomly divided into three groups: control (CTL; n=9), Normal Interval without Kaatsu (NIWK; n=9), and Interval with Kaatsu (IWK; n=10). Pre-tests were measured at beginning of exercise protocol. Subjects of current research were well trained football player and without chronic injuries.

Seven steps Bruce protocol was used for determining of aerobic capacity and maximum oxygen uptake (Sarma \& Levine, 2016). Participants on this study spent minimum 10 minutes warm-up before start the test. Speed and slope of treadmill gradually increased. Participants were allowed to stop test when they could not continue test (Time to exhaustion was recorede). Metalyzer 3B device (Meyer, Georg, Becker, \& Kindermann, 2001), Metasoft Studio software and COSMOD treadmill were applied for aerobic test protocol. Devices were calibrated prior data collection and before post-test. Volume of Vo2 and Vco2 was measured directly by device. Heart rate was monitored by Polar chest belt which was connected to Metalyzer. All tests were performed at laboratory of Shomal University with standard temperature. 
Rate of Perceived Exertion (RPE) were determined by 15 point Borg Scale at the end of step 3 during Bruce protocol (Ciolac et al., 2015). Subjects were participated at familiar session 48 hours before start the test. All subjects were familiar with procedure of Bruce Protocol prior the experimental began.

In the first session of the exercise for the purpose of body adaptation and avoiding any injuries, the imposed pressure was $140 \mathrm{mmHG}$ and to reach the aimed pressure, from the second session the pressure was increased to $180 \mathrm{mmHG}$ (Scott, Loenneke, Slattery, \& Dascombe, 2015). Adjustable cuff was applied for blood restriction protocols. The cuffs were applied tightly around the upper section of thigh (One cuff was used for per subjects during exercise protocols).

\begin{tabular}{ccccc}
\multicolumn{4}{c}{ TABLE 1 Exercise Protocol } & \\
Week & Session & Set & Intensity & Distance \\
\hline First & 4 & 3 & $60-65 \%$ MHRR & $400 \mathrm{~m}$ \\
Second & 4 & 4 & $65-70 \%$ MHRR & $400 \mathrm{~m}$ \\
\hline
\end{tabular}

Intermediate intensity interval training (aerobic energy system exercise) was applied for exercise protocol. Three set at $400 \mathrm{~m}$ for the first week and 4 set $(400 \mathrm{~m})$ for the second week including $60-80$ seconds rest between sets were applied in this experimental. Warm-up and cool-down were done at initial and end of each training session. Training intensity was monitored by Polar base chest belt and windows client software. Intensity of training was selected at 60-70\% maximum heart rate reserve (MHRR).

\section{Statistical analysis}

Analyses of variance with one-way ANOVA was applied to determination of between groups' differences and in the case of significant $\mathrm{F}$ value, a post hoc test using Bonferroni identified significant differences among mean values between groups. Paired t-test was used to determination of variables changes within the groups from pre to post test. Kolmogorov-Smirnov was applied for determination of normality of the groups. The level of significance was established a priori as $\mathrm{p} \leq 0.05$. SPSS software was employed to analyze the data.

\section{Results}

The result of statistical analysis has been shown there was a significant differences of rate of perceive exertion at the $\mathrm{p}<.05$ level for the three groups $[\mathrm{F}(2,25)=7.243, \mathrm{p}=0.003]$. Post hoc comparison using by bonferroni indicated that the score of RPE at control group was significantly different that the IWK and NIWK group.

\section{$\square$ Mean $\square$ SD}

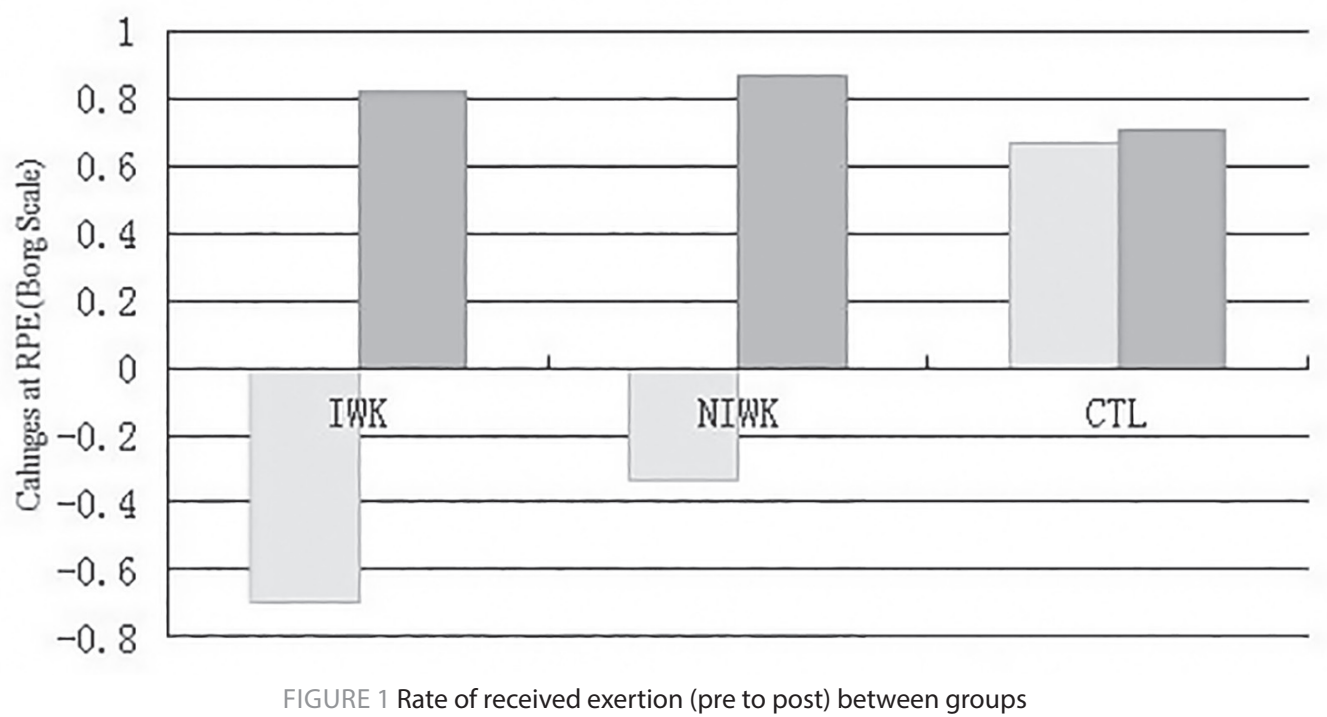

Did not significantly differ from the NIWK and IWK groups. Analyzing data from pre to posttest using by paired $\mathrm{T}$ test has been shown a significant differences in the score of RPE from pre to post test at IWK group; $\mathrm{t}(9)=2.689, \mathrm{p}=0.025$ and CTL group; $\mathrm{t}(9)=-2.828, \mathrm{p}=0.022$. Not any significant differences reported at NIWK group (Figure 1).

TABLE 2 Deceptive Data for Pre to Post Tests

\begin{tabular}{|c|c|c|c|c|c|c|}
\hline \multirow[t]{2}{*}{ Variable } & \multicolumn{2}{|c|}{ Control group } & \multicolumn{2}{|c|}{ Kaatsu group } & \multicolumn{2}{|c|}{ Normal Interval } \\
\hline & pretest & post test & pretest & post test & pretest & post test \\
\hline RPE & $15.88 \pm 1.26$ & $16.55 \pm 1.23$ & $15.2 \pm 1.75$ & $14.5 \pm 1.08$ & $15.0 \pm 1.87$ & $14.66 \pm 1.5$ \\
\hline VO2max & $52.22 \pm 5.04$ & $50.22 \pm 5.4$ & $54.6 \pm 6.99$ & $56.6 \pm 7.74$ & $54.11 \pm 5.66$ & $54.88 \pm 5.68$ \\
\hline
\end{tabular}


The result of statistical analysis has been shown there was a significant differences of maximum oxygen uptake $\left(\mathrm{VO}_{2} \max \right)$ at the $\mathrm{p}<.05$ level for the three groups $[\mathrm{F}(2,25)=13.349, \mathrm{p}=0.000]$. Post hoc comparison using by bonferroni indicated that the score of $\mathrm{VO}_{2} \max$ at control group was significantly different that the IWK and NIWK group. Did not significantly differ from the NIWK and IWK groups. Analyzing data from pre to posttest using by paired $\mathrm{T}$ test has been shown a significant differences in the score of maximum oxygen uptake from pre to post test at IWK group; $t(9)=-3.721, p=0.005$ and CTL group; $t(9)=3.464, p=0.009$. Not any significant differences reported at NIWK group (Figure 2).

\section{$\square$ Mean $\square S D$}



FIGURE 2 Comparison of change at VO2max (pre to post) between groups

\section{Discussion}

The strong relationship between training intensity maximum oxygen uptake reported at the several studies. It has been shown that any reducing at training intensity my lead the maximum oxygen uptake to lower than normal (Kenney, Wilmore, \& Costill, 2015). It has been reported decreasing at maximum oxygen uptake by result of reducing training intensity and volume at the transition phases of periodization in football. Increasing intensity of aerobic energy system training with some other way without manipulating actual volume and intensity factors such as distance and speed with minimum side effect on recovery and fatigue by result of completion phase may help researcher to minimizing at $\mathrm{VO}_{2}$ max reduction (Shepley et al., 1992). This study investigated effect of interval training (moderate intensity-aerobic energy system) with blood flow restriction on the aerobic performance and rate of perceived exertion. Cuff pressure between 140-180mHg was applied at this research. Several investigation previously reported benefits of KATTSU training on the strength training by increasing muscle mass and one repetition maximum but still effect of this type of training on the aerobic performance and related variables is not clear yet. The result of this research has been shown improving maximum oxygen uptake by result of blood restriction training. The present study has been shown significant differences at maximum oxygen uptake between groups. It has been estimated increasing $3.66 \%$ at $\mathrm{VO}_{2} \max$ in interval groups under blood restriction while has been shown only $1.43 \%$ increasing at maximum oxygen uptake in interval groups without blood restriction. In additional to the control groups $-3.82 \%$ decreasing of $\mathrm{VO}_{2}$ max has been reported (reported by paired $t$ test for within group differences). The magnitude of increases in $\mathrm{VO}_{2}$ max at KAATSU group in current study is also similar to those reported by Park et al., that they investigated walking training with blood flow restriction (Park et al., 2010). It Has been reported increasing $2.5 \%$ at aerobic capacity after two weeks walking training. The similar finding has been
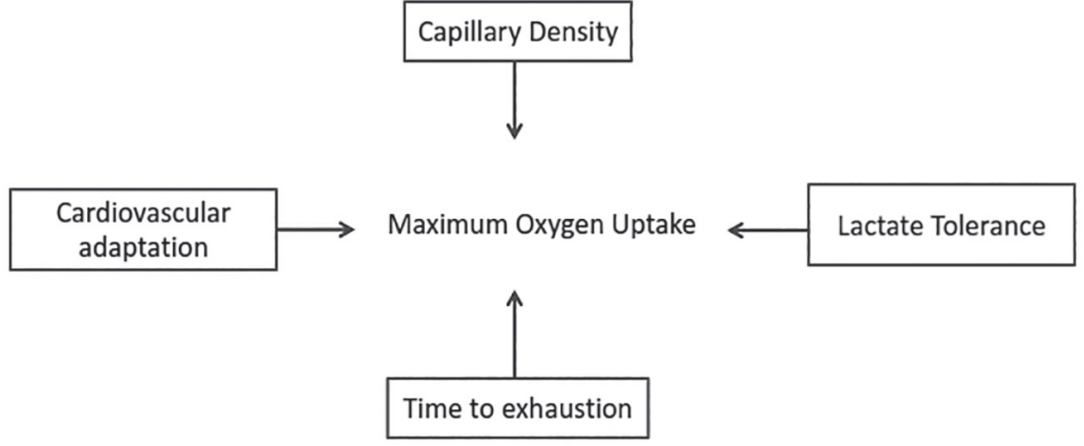

FIGURE 3 Suggested model in variables relationship with maximum oxygen uptake 
reported by Takashi et al. They has been reported improving by $6.4 \%$ at $\mathrm{VO}_{2}$ max after 8 weeks training with intensity of $40 \%$ of $\mathrm{VO}_{2} \max$ (Abe et al., 2010). Result of current research also has been shown improving time to exhaustion among kaatsu groups and rate of perceived exertion. Rate of perceived exertion is effective variable for endurance and aerobic performance. Improving capillary density (Patterson \& Ferguson, 2010), developing time to fatigue, improving cardiovascular function (Ozaki et al., 2010), respiratory function and muscular performance may are the main factors by resulted by blood restriction training to improving maximum oxygen uptake and aerobic performance. This research suggested model of improving aerobic performance by blood flow restriction (Figure 3) which need more research at feature to show all aspects by details. The findings from this study make several contributions to the current literature. First, improving maximum oxygen uptake without manipulating of distance and speed of training (factors of Intensity during aerobic energy system training) with blood flow restriction method. This is the first time report at this area.

The major limitation of this study was small sample of participants, and future study might explore in larger sample. The second limitation was level of participants of the current study.

In conclusion, our investigation in the current research suggests that moderate-intensity aerobic exercise (running) may improve aerobic performance in combined with blood flow restriction method by improving rate of perceived exertion and maximum oxygen uptake at comparison with traditional training.

\section{Acknowledgments}

The authors gratefully acknowledge all the help of the football player who participated in this study. Our special appreciation goes to all the laboratory staff of Department of sports physiology in Shomal University for their kindness and support during the research work.

\section{REFERENCES}

Abe, T., Sakamaki, M., Fujita, S., Ozaki, H., Sugaya, M., Sato, Y., \& Nakajima, T. (2010). Effects of LowIntensity Walk Training With Restricted Leg Blood Flow on Muscle Strength and Aerobic Capacity in Older Adults. Journal of Geriatric Physical Therapy, 33(1), 34-40. doi: 10.1097/JPT.0b013e3181d07a73

Bangsbo, J. (2003). Physiology of training. Science and soccer, 2, 47-58.

Behi, A., Fahey, T. D., Afsharnezhad, T., \& Amani, A. R. (2017). Effect of High Intensity Interval Training with Blood Restriction on Anaerobic Performance. International Journal of Applied Exercise Physiology, $6(2), 45-52$

Benson, R., \& Connolly, D. (2011). Heart rate training: Champaign, IL: Human Kinetics.

Ciolac, E. G., Mantuani, S. S., Neiva, C. M., Verardi, C. E. L., Pêssoa-Filho, D. M., \& Pimenta, L. (2015). Rating of perceived exertion as a tool for prescribing and self regulating interval training: a pilot study. Biology of sport, 32(2), 103.

Clemente, F. M., Couceiro, M. S., Martins, L., Manuel, F., Ivanova, M. O., \& Mendes, R. (2013). Activity profiles of soccer players during the 2010 world cup. Journal of Human Kinetics, 38, 201-211.

Cook, S. B., Clark, B. C., \& Ploutz-Snyder, L. L. (2007). Effects of Exercise Load and Blood-Flow Restriction on Skeletal Muscle Function. Medicine \& Science in Sports \& Exercise, 39(10), 1708-1713. doi: 10.1249/ mss.0b013e31812383d6

Evans, C., Vance, S., \& Brown, M. (2010). Short-term resistance training with blood flow restriction enhances microvascular filtration capacity of human calf muscles. Journal of Sports Sciences, 28(9), 999-1007.

Gibala, M. J., Little, J. P., Van Essen, M., Wilkin, G. P., Burgomaster, K. A., Safdar, A., . . Tarnopolsky, M. A. (2006). Short-term sprint interval versus traditional endurance training: similar initial adaptations in human skeletal muscle and exercise performance. The Journal of physiology, 575(3), 901-911.

Gilman, M. B. (1996). The use of heart rate to monitor the intensity of endurance training. Sports Medicine, 21(2), 73-79.

Helgerud, J., Engen, L. C., Wisløff, U., \& Hoff, J. (2001). Aerobic endurance training improves soccer performance. Medicine \& Science in Sports \& Exercise, 33(11), 1925-1931.

Helgerud, J., Høydal, K., Wang, E., Karlsen, T., Berg, P., Bjerkaas, M., . . . Bach, R. (2007). Aerobic highintensity intervals improve V O2max more than moderate training. Medicine \& Science in Sports \& Exercise, 39(4), 665-671.

Kenney, W. L., Wilmore, J., \& Costill, D. (2015). Physiology of Sport and Exercise 6th Edition: Champaign, IL: Human Kinetics.

Kirkendall, D. T., \& Garrett, W. E. (1998). The effects of aging and training on skeletal muscle. The American Journal of Sports Medicine, 26(4), 598-602.

Loenneke, J. P., Wilson, G. J., \& Wilson, J. M. (2010). A Mechanistic Approach to Blood Flow Occlusion. Int J Sports Med, 31(01), 1-4. doi: 10.1055/s-0029-1239499

Meyer, T., Georg, T., Becker, C., \& Kindermann, W. (2001). Reliability of gas exchange measurements from two different spiroergometry systems. International journal of sports medicine, 22(08), 593-597.

Ozaki, H., Sakamaki, M., Yasuda, T., Fujita, S., Ogasawara, R., Sugaya, M., ... Abe, T. (2010). Increases in thigh muscle volume and strength by walk training with leg blood flow reduction in older participants. Journals of Gerontology Series A: Biomedical Sciences and Medical Sciences, 66(3), 257-263.

Park, S., Kim, J. K., Choi, H. M., Kim, H. G., Beekley, M. D., \& Nho, H. (2010). Increase in maximal oxygen uptake following 2-week walk training with blood flow occlusion in athletes. European Journal of Applied 
Physiology, 109(4), 591-600. doi: 10.1007/s00421-010-1377-y

Patterson, S. D., \& Ferguson, R. A. (2010). Increase in calf post-occlusive blood flow and strength following short-term resistance exercise training with blood flow restriction in young women. European Journal of Applied Physiology, 108(5), 1025-1033.

Presta, M., Dell'Era, P., Mitola, S., Moroni, E., Ronca, R., \& Rusnati, M. (2005). Fibroblast growth factor/ fibroblast growth factor receptor system in angiogenesis. Cytokine \& growth factor reviews, 16(2), 159-178.

Rivera-Brown, A. M., \& Frontera, W. R. (2012). Principles of exercise physiology: responses to acute exercise and long-term adaptations to training. $P M \nLeftarrow R, 4(11), 797-804$.

Sarma, S., \& Levine, B. D. (2016). Beyond the Bruce Protocol. Cardiology clinics, 34(4), 603-608.

Scherr, J., Wolfarth, B., Christle, J. W., Pressler, A., Wagenpfeil, S., \& Halle, M. (2013). Associations between Borg's rating of perceived exertion and physiological measures of exercise intensity. European Journal of Applied Physiology, 113(1), 147-155.

Scott, B. R., Loenneke, J. P., Slattery, K. M., \& Dascombe, B. J. (2015). Exercise with blood flow restriction: an updated evidence-based approach for enhanced muscular development. Sports Medicine, 45(3), 313-325.

Shepley, B., MacDougall, J. D., Cipriano, N., Sutton, J. R., Tarnopolsky, M. A., \& Coates, G. (1992). Physiological effects of tapering in highly trained athletes. Journal of Applied Physiology, 72(2), 706-711.

Weston, A. R., Myburgh, K. H., Lindsay, F. H., Dennis, S. C., Noakes, T. D., \& Hawley, J. A. (1996). Skeletal muscle buffering capacity and endurance performance after high-intensity interval training by welltrained cyclists. European journal of applied physiology and occupational physiology, 75(1), 7-13. 